\title{
On the GLR and UMP tests in the family with support dependent on the parameter
}

\author{
Abbas Eftekharian ${ }^{1}$, Seyed Mahmoud Taheri ${ }^{2}, *$ \\ ${ }^{1}$ Department of Statistics, School of Science, Hormozgan University, Bandar Abbas, Iran. \\ ${ }^{2}$ Faculty of Engineering Science, College of Engineering, University of Tehran, Tehran, Iran.
}

Received: 28 September 2014; Accepted: 27 June 2015

Editor: Rahim Mahmoudvand

\begin{abstract}
Some general results about the GLR tests, for testing simple hypothesis versus two-sided hypothesis, in the family with support dependent on the parameter, are obtained. In addition, we show that such GLR tests are equivalent to the UMP tests in the same problems. Moreover, we derive the general form of the UMP tests for testing an interval hypothesis versus two-sided alternative.
\end{abstract}

Keywords Uniformly most powerful test, Generalized likelihood ratio test, Two-sided hypothesis.

AMS 2010 subject classifications 62F03

DOI: $10.19139 /$ soic.v3i3.107

\section{Introduction and Background}

The problem of finding uniformly most powerful (UMP) test for testing the simple hypothesis $H_{0}: \theta=\theta_{0}$ against two-sided alternative $H_{1}: \theta \neq \theta_{0}$ has been an interesting problem in testing statistical hypotheses. It is well known that, for distributions belong to the exponential family, such UMP tests do not exist (see [7]). On the other hand, there has been some attempts to find UMP tests for other distributions and/or for the problem of testing one-sided hypotheses. Historically, studies on UMP tests started in the 1940's. Lehmann and Stein [6] presented some most powerful tests for the parameter of a normal distribution. Birnbaum [3] and Pratt [12] offered the solutions for UMP tests for composite hypotheses in the uniform distributions with both end points of the support dependent on the parameter. Takeuchi [15] constructed UMP test for the location parameter of an exponential distribution despite the existence of a nuisance parameter. Kabe and Laurent [5] exhibited a UMP test for a two-sided hypothesis in the double exponential distribution with a nuisance parameter. Edelman [4] showed that the usual two-sided test is actually uniformly most powerful within the class of tests with critical regions that are symmetric about the null hypothesis. Nomakuchi [11] studied the UMP tests in the presence of a nuisance parameter for uniform distribution. A new method to determine UMP tests in discrete sample spaces was proposed by Scherb [14]. Wang et al. [16] constructed a UMPU test for comparing the means of two negative binomial distributions. Migliorati [10] demonstrated that a UMP test exists for testing two-sided hypothesis in the family with support dependent on the parameter. Also, McDermott and Wang [9] investigated a construction of uniformly more powerful tests for hypotheses about linear inequalities. Aaberge and Zhang [1] presented a class of exact UMP unbiased tests

\footnotetext{
${ }^{*}$ Correspondence to: Seyed Mahmoud Taheri (Email: sm_taheri@ut.ac.ir). Faculty of Engineering Science, College of Engineering,
} University of Tehran, Tehran, Iran.

ISSN 2310-5070 (online) ISSN 2311-004X (print)

Copyright (C) 2015 International Academic Press 
for testing conditional symmetry against various alternative diagonals-parameter. The UMP two-sided test in the family with right and left extreme points of the support dependent on the parameter is obtained by Sayyareh et al. [13].

But, as far as the authors know, there has been neither general result about the existence and formulation of the two-sided generalized likelihood ratio (GLR) test in the family with support dependent to parameter, nor about the formal relationship between such tests and the related UMP tests in this family.

In this paper, we obtain the GLR tests for testing hypothesis $H_{0}: \theta=\theta_{0}$ versus two-sided alternative $H_{1}: \theta \neq$ $\theta_{0}$, in the family of distributions of the following forms

$$
f_{\theta}(x)=a(\theta) b(x) \quad ; \quad c<x<\theta, a(\theta)>0,
$$

and

$$
g_{\theta}(x)=p(\theta) q(x) \quad ; \quad \theta<x<d, p(\theta)>0 .
$$

We also demonstrate that UMP tests for testing the above hypotheses are equivalent to the GLR tests. In addition, we derive the general form of UMP tests for testing $H_{0}: \theta_{1} \leq \theta \leq \theta_{2}$ versus $H_{1}: \theta<\theta_{1}$ or $\theta>\theta_{2}$ in the families with the right or the left extreme point of the support dependent on the parameter.

This paper is organized as follows: In Section 2, we obtain the general form of the GLR test for testing simple hypothesis versus two-sided alternative in the family with the right or left extreme point of the support dependent on the parameter. In Section 3, we obtain the UMP test for testing $H_{0}: \theta_{1} \leq \theta \leq \theta_{2}$ versus $H_{1}: \theta<\theta_{1}$ or $\theta>\theta_{2}$ in the families with the right or the left extreme point of the support dependent on the parameter.

In the following, we will use the notations $X_{(1)}$ for $\min \left(X_{1}, \ldots, X_{n}\right)$, and $X_{(n)}$ for $\max \left(X_{1}, \ldots, X_{n}\right)$.

\section{GLR two-sided test in the family with right (left) extreme point of the support dependent on the parameter}

Let us begin this section with a Lemma.

\section{Lemma 1}

i) If

$$
f_{\theta}(x)=a(\theta) b(x) \quad ; \quad c<x<\theta, a(\theta)>0,
$$

be a continuous function, then $a(\cdot)$ is a decreasing function.

ii) If

$$
g_{\theta}(x)=p(\theta) q(x) \quad ; \quad \theta<x<d, p(\theta)>0,
$$

be a continuous function, then $p(\cdot)$ is an increasing function.

Proof

i) $\int_{c}^{\theta} a(\theta) b(x) d x=1$, implies that $\int_{c}^{\theta} b(x) d x=1 / a(\theta)$. Now we differentiate from both sided of the second relation to obtain $b(\theta)=-a^{\prime}(\theta) /(a(\theta))^{2}$. But $b(\cdot)$ is a nonnegative function, therefore $a(\cdot)$ is a decreasing function.

ii) The proof can be done in a similar way to the case (i).

Now, we obtain the general forms of the GLR tests for testing hypothesis $H_{0}: \theta=\theta_{0}$ versus $H_{1}: \theta \neq \theta_{0}$ in families with density (1) and (2).

\section{Theorem 1}

Suppose that $\underline{X}=\left(X_{1}, X_{2}, \ldots, X_{n}\right)$ is a random sample, with observed value $\underline{x}=\left(x_{1}, x_{2}, \ldots, x_{n}\right)$, from a distribution with density function (1). Then a GLR test of size $\alpha$ for testing $H_{0}: \theta=\theta_{0}$ versus $H_{1}: \theta \neq \theta_{0}$ is given by

$$
\psi_{1}^{*}(\underline{x})=\left\{\begin{array}{cc}
1 & x_{(n)}>\theta_{0} \text { or } x_{(n)}<a^{-1}\left(a\left(\theta_{0}\right) \alpha^{-\frac{1}{n}}\right), \\
0 & \text { otherwise. }
\end{array}\right.
$$


Proof

Let $\Theta=\{\theta: \theta>c\}, \Theta_{0}=\left\{\theta: \theta=\theta_{0}\right\}$

$$
L(\theta ; \underline{x})=(a(\theta))^{n} \prod_{i=1}^{n} b\left(x_{i}\right) I_{(c, \theta)}\left(x_{i}\right)=\left\{\begin{array}{cc}
(a(\theta))^{n} \prod_{i=1}^{n} b\left(x_{i}\right) & x_{(n)} \leq \theta \\
0 & x_{(n)}>\theta
\end{array}\right.
$$

It is obvious that, $\operatorname{MLE}(\theta)_{\theta \in \Theta}=X_{(n)}$ and $\operatorname{MLE}(\theta)_{\theta \in \Theta_{0}}=\theta_{0}$, and

$$
\lambda(\underline{x})=\frac{\sup _{\Theta_{0}} L(\theta ; \underline{x})}{\sup _{\Theta} L(\theta ; \underline{x})}=\frac{\left(a\left(\theta_{0}\right)\right)^{n} \prod_{i=1}^{n} b\left(x_{i}\right)}{\left(a\left(x_{(n)}\right)\right)^{n} \prod_{i=1}^{n} b\left(x_{i}\right)}=\left\{\begin{array}{cl}
\left(\frac{a\left(\theta_{0}\right)}{a\left(x_{(n))}\right)}\right)^{n} & x_{(n)} \leq \theta_{0}, \\
0 & x_{(n)}>\theta_{0} .
\end{array}\right.
$$

So, the GLR test rejects $H_{0}$, iff $x_{(n)}>\theta_{0}$ or $\left(a\left(\theta_{0}\right) / a\left(x_{(n)}\right)\right)^{n} \leq k_{\alpha}$ when $x_{(n)} \leq \theta_{0}$; and iff $x_{(n)}>\theta_{0}$ or $x_{(n)}<k_{\alpha}^{\prime}$ when $x_{(n)} \leq \theta_{0}$, where $k_{\alpha}^{\prime}$ is chosen so that $P_{\theta_{0}}\left(X_{(n)}<k_{\alpha}^{\prime}\right)=\alpha$. Using Lemma 1 and distribution of $X_{(n)}$, we have

$$
\alpha=\int_{c}^{k_{\alpha}^{\prime}} n b(t) \frac{\left(a\left(\theta_{0}\right)\right)^{n}}{(a(t))^{n-1}} d t, \quad k_{\alpha}^{\prime}=a^{-1}\left(a\left(\theta_{0}\right) \alpha^{-\frac{1}{n}}\right) .
$$

Hence the GLR test rejects $H_{0}$ iff $X_{(n)}<a^{-1}\left(a\left(\theta_{0}\right) \alpha^{-\frac{1}{n}}\right)$ for $X_{(n)} \leq \theta_{0}$ or $X_{(n)}>\theta_{0}$.

\section{Theorem 2}

Suppose that $\underline{X}=\left(X_{1}, X_{2}, \ldots, X_{n}\right)$ is a random sample, with observed value $\underline{x}=\left(x_{1}, x_{2}, \ldots, x_{n}\right)$, from a distribution with density function (2). Then a GLR test of size $\alpha$ for the simple hypothesis $H_{0}: \theta=\theta_{0}$ versus two-sided alternative $H_{1}: \theta \neq \theta_{0}$ is given by

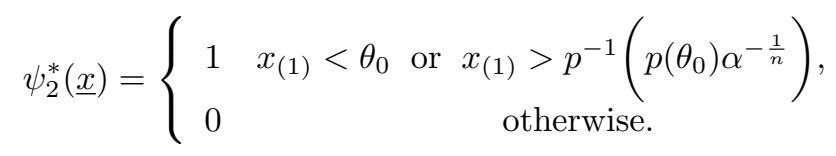

Proof

The proof is similar to the proof of Theorem 1 .

\section{Example 1}

Let $\underline{X}=\left(X_{1}, X_{2}, \ldots, X_{n}\right)$ is a random sample, with observed value $\underline{x}=\left(x_{1}, x_{2}, \ldots, x_{n}\right)$, from a distribution with density function

$$
f_{\theta}(x)=\frac{2 x}{\theta^{2}} \quad ; \quad 0<x<\theta .
$$

The GLR test of size $\alpha$ for hypothesis $H_{0}: \theta=\theta_{0}$ versus $H_{1}: \theta \neq \theta_{0}$ is given by

$$
\psi^{*}(\underline{x})=\left\{\begin{array}{cc}
1 & x_{(n)}>\theta_{0} \text { or } x_{(n)}<\theta_{0} \alpha^{\frac{1}{2 n}} \\
0 & \text { otherwise. }
\end{array}\right.
$$

\section{Remark 1}

It is shown by Birkes [2] that, in the one-parameter exponential family the GLR test for testing one-sided hypotheses coincides with the UMP test. He also shown that in this family under certain conditions on the likelihood function, GLR test for testing a two-sided hypothesis is usually coincide with the UMPU test. But as the authors know, there has been no works on the relationship between UMP tests and GLR tests for testing a simple versus two-sided hypotheses.

\section{Remark 2}

The GLR tests obtained in Theorems 1 and 2 are coincided with the UMP tests in the same problems (see Theorems 4.3 and 4.4 in [13]). 


\section{UMP test for testing composite hypotheses}

In this section, we will acquire UMP tests for testing hypothesis $H_{0}: \theta_{1} \leq \theta \leq \theta_{2}$ versus $H_{1}: \theta<\theta_{1}$ or $\theta>\theta_{2}$ in the family with support dependent on the parameter. Note that, such tests don't exist for the exponential family, but it is shown that there exist a level $\alpha$ UMPU test for such hypotheses for one-parameter exponential family (see [7]).

\section{Theorem 3}

Suppose that $\underline{X}=\left(X_{1}, X_{2}, \ldots, X_{n}\right)$ is a random sample, with observed value $\underline{x}=\left(x_{1}, x_{2}, \ldots, x_{n}\right)$, from a distribution with density function (1). Then a UMP test of size $\alpha$ for a hypothesis $H_{0}: \theta_{1} \leq \theta \leq \theta_{2}$ versus $H_{1}: \theta<\theta_{1}$ or $\theta>\theta_{2}$ is given by

$$
\phi^{*}(\underline{x})=\left\{\begin{array}{cc}
1 & x_{(n)} \leq k_{1} \text { or } x_{(n)}>k_{2} \\
0 & \text { otherwise }
\end{array}\right.
$$

where $k_{1}=a^{-1}\left(a\left(\theta_{1}\right) \alpha^{-\frac{1}{n}}\right)$ and $k_{2}=a^{-1}\left(a\left(\theta_{2}\right)\left\{1-\alpha\left(1-\left(\frac{a\left(\theta_{2}\right)}{a\left(\theta_{1}\right)}\right)^{n}\right)\right\}^{-\frac{1}{n}}\right)$ are chosen so that $\alpha=$ $E_{\theta_{1}}\left[\phi^{*}(\underline{X})\right]=E_{\theta_{2}}\left[\phi^{*}(\underline{X})\right]$.

Proof

First we divide $H_{1}$ into $H_{1}^{\prime}: \theta<\theta_{1}$ and $H_{1}^{\prime \prime}: \theta>\theta_{2}$. Then we find a UMP test for $H_{1}^{\prime}$ and $H_{1}^{\prime \prime}$.

1. Consider the problem of testing hypotheses

$$
\left\{\begin{array}{l}
H_{0}: \theta \geq \theta_{1},\left(\theta_{1}<\theta_{2}\right) \\
H_{1}^{\prime}: \theta<\theta_{1}
\end{array}\right.
$$

Since the probability density function (1) has the monotone likelihood ratio at $X_{(n)}$, so a UMP test for (4) is as follows

$$
\phi_{1}(\underline{x})= \begin{cases}1 & x_{(n)} \leq k_{1} \\ 0 & x_{(n)}>k_{1}\end{cases}
$$

which is a UMP test of size $\alpha$ on the condition that $\alpha=E_{\theta_{1}}\left[\phi_{1}(\underline{X})\right]$. Meanwhile it is easy to show that $k_{1}=a^{-1}\left(a\left(\theta_{1}\right) \alpha^{-\frac{1}{n}}\right)$. Therefore, the power function of $\phi_{1}$ is as follows

$$
\begin{aligned}
\pi_{\phi_{1}}(\theta) & =P_{\theta}\left(X_{(n)}<k_{1}\right) \\
& = \begin{cases}1 & \theta \leq k_{1}, \\
\alpha\left(\frac{a(\theta)}{a\left(\theta_{1}\right)}\right)^{n} & \theta>k_{1} .\end{cases}
\end{aligned}
$$

2. Now, we find a UMP for

$$
\left\{\begin{array}{l}
H_{0}: \theta=\theta_{0} \\
H_{1}^{\prime \prime}: \theta>\theta_{2}>\theta_{0}
\end{array}\right.
$$

where $\theta_{0} \in\left[\theta_{1}, \theta_{2}\right]$. For this purpose, first consider the problem of testing the following simple hypotheses

$$
\left\{\begin{array}{l}
H_{0}: \theta=\theta_{0} \\
H_{12}^{\prime \prime}: \theta=\theta_{2}^{\prime}
\end{array}\left(\theta_{0} \leq \theta_{2}<\theta_{2}^{\prime}\right) .\right.
$$

According to Neyman-Pearson Lemma, each test conforming with

$$
\phi_{2}(\underline{x})= \begin{cases}1 & r(\underline{x})>k_{2} \\ \gamma & r(\underline{x})=k_{2} \\ 0 & r(\underline{x})<k_{2}\end{cases}
$$


is a MP test of size $\alpha$ for the hypotheses (6) on the condition that (see [7])

$$
\alpha=E_{\theta_{0}}\left[\phi_{2}(\underline{X})\right]=P_{\theta_{0}}\left(r(\underline{X})>k_{2}\right)+\gamma P_{\theta_{0}}\left(r(\underline{X})=k_{2}\right),
$$

where

$$
r(\underline{x})=\frac{f_{\theta_{2}^{\prime}}(\underline{x})}{f_{\theta_{0}}(\underline{x})}=\left(\frac{a\left(\theta_{2}^{\prime}\right)}{a\left(\theta_{0}\right)}\right)^{n} \frac{I_{\left(c, \theta_{2}^{\prime}\right)}\left(x_{(n)}\right)}{I_{\left(c, \theta_{0}\right)}\left(x_{(n)}\right)} .
$$

On the other hand, we know that $\theta_{0} \in\left[\theta_{1}, \theta_{2}\right]$, so we can choose each value in this interval for $\theta_{0}$. Now, let $\theta_{0}=\theta_{2}$, thus

$$
r(\underline{x})=\left\{\begin{array}{cl}
\left(\frac{a\left(\theta_{2}^{\prime}\right)}{a\left(\theta_{0}\right)}\right)^{n} & x_{(n)} \leq \theta_{0}, \\
\infty & x_{(n)}>\theta_{0} .
\end{array}\right.
$$

It is clear that $r(\underline{x})$ is increasing with respect to $x_{(n)}$. So, $\phi_{2}$ consists of

$$
\phi_{2}(\underline{x})= \begin{cases}1 & x_{(n)}>k_{2} \\ 0 & x_{(n)} \leq k_{2}\end{cases}
$$

is a MP test of size $\alpha$ for the related hypotheses. But, $\phi_{2}$ depends on $\theta_{2}^{\prime}$ only by $\theta_{0} \leq \theta_{2}<\theta_{2}^{\prime}$, thus $\phi_{2}$ is a UMP test of size $\alpha$ for testing hypotheses (5) when

$$
\alpha=E_{\theta_{2}}\left[\phi_{2}(\underline{X})\right] .
$$

It can be shown that the power function of $\phi_{2}$ is given by

$$
\pi_{\phi_{2}}(\theta)=1-(1-\alpha)\left(\frac{a(\theta)}{a\left(\theta_{2}\right)}\right)^{n}, \quad \theta \geq k_{2} .
$$

Now, it is seen that $\phi^{*}(\underline{x})$ is a UMP test of size $\alpha$ for testing the hypothesis $H_{0}: \theta_{1} \leq \theta \leq \theta_{2}$ versus $H_{1}: \theta<$ $\theta_{1}$ or $\theta>\theta_{2}$. Moreover, the power function of $\phi^{*}$ is given by

$$
\begin{aligned}
\pi_{\phi^{*}}(\theta)= & P_{\theta}\left(X_{(n)}<k_{1}\right)+P_{\theta}\left(X_{(n)}>k_{2}\right) \\
= & \begin{cases}1 & \theta<k_{1}, \\
\alpha\left(\frac{a(\theta)}{a\left(\theta_{1}\right)}\right)^{n} & k_{1} \leq \theta<k_{2}, \\
1-(1-\alpha)\left(\frac{a(\theta)}{a\left(\theta_{2}\right)}\right)^{n} & \theta \geq k_{2} .\end{cases}
\end{aligned}
$$

\section{Theorem 4}

Suppose that $\underline{X}=\left(X_{1}, X_{2}, \ldots, X_{n}\right)$ is a random sample, with observed value $\underline{x}=\left(x_{1}, x_{2}, \ldots, x_{n}\right)$, from a distribution with density function (2). Then a UMP test of size $\alpha$ for a hypothesis $H_{0}: \theta_{1} \leq \theta \leq \theta_{2}$ versus $H_{1}: \theta<\theta_{1}$ or $\theta>\theta_{2}$ is given by

$$
\varphi^{*}(\underline{x})=\left\{\begin{array}{cc}
1 & x_{(1)}<m_{1} \text { or } x_{(1)} \geq m_{2}, \\
0 & \text { otherwise }
\end{array}\right.
$$

where $m_{1}$ and $m_{2}$ are chosen so that $\alpha=E_{\theta_{1}}\left[\varphi^{*}(\underline{X})\right]=E_{\theta_{2}}\left[\varphi^{*}(\underline{X})\right]$.

Proof

The proof is similar to the proof of Theorem 3. 


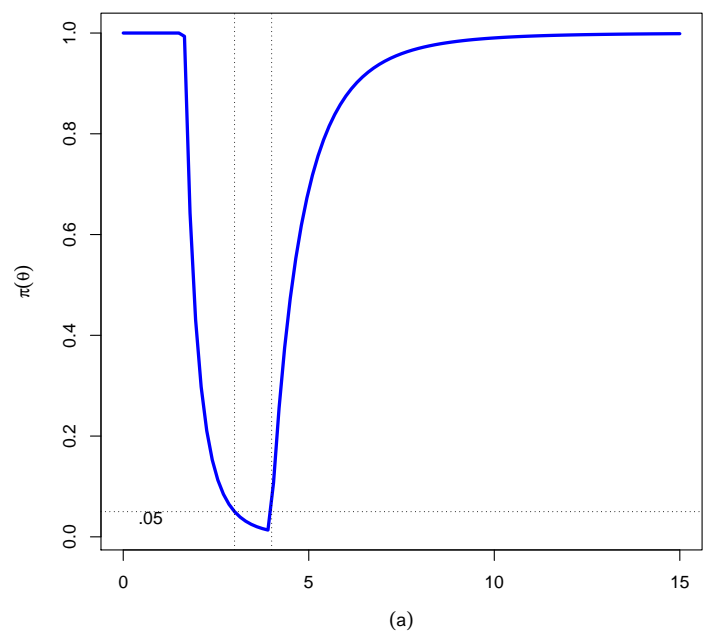

(a) $n=5$ and $\alpha=0.05$.

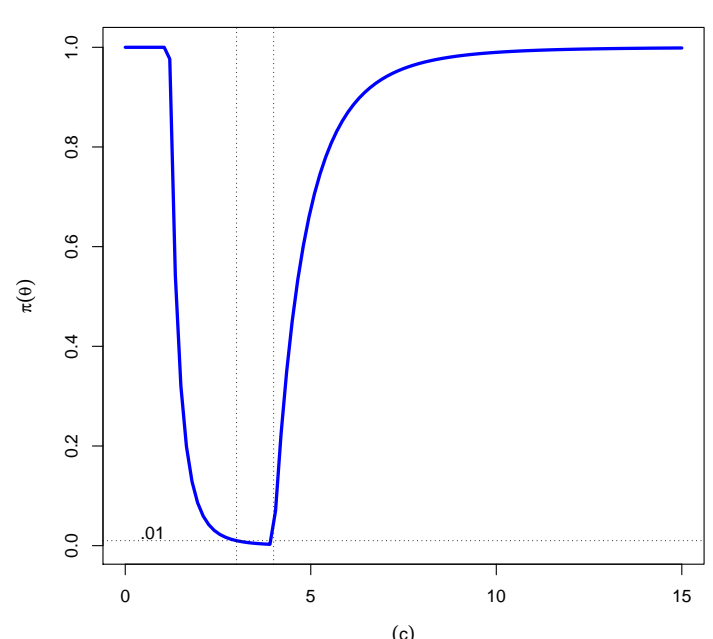

(c) $n=5$ and $\alpha=0.01$.

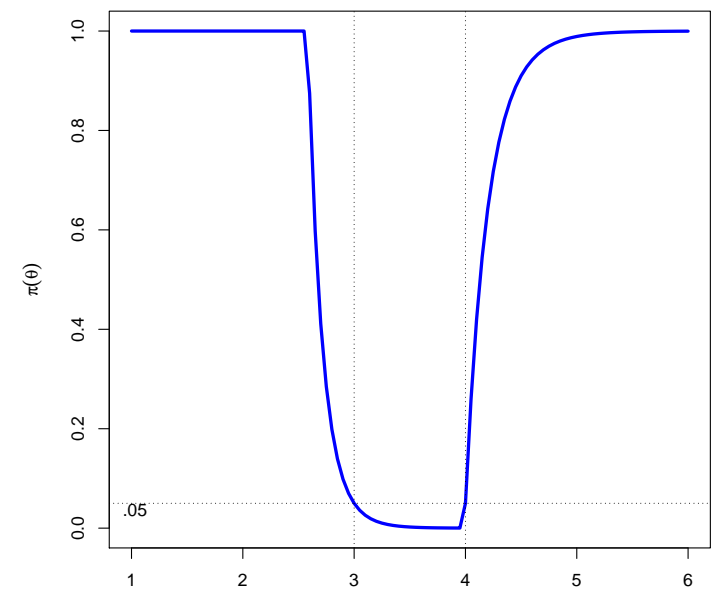

(b)

(b) $n=20$ and $\alpha=0.05$.

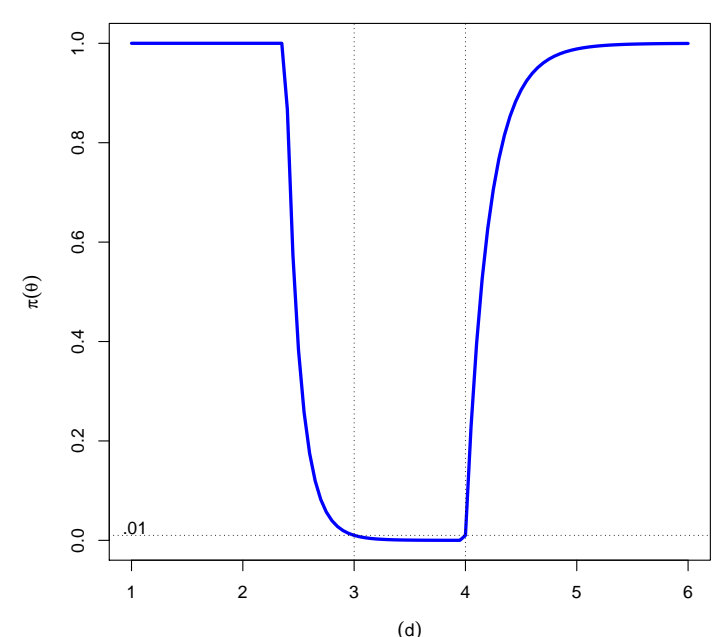

(d) $n=20$ and $\alpha=0.01$.

Figure 1. The curves of $\pi_{\phi^{*}}(\theta)$ in Example 2.

\section{Remark 3}

If in Theorems 3 and $4, \theta_{1}=\theta_{2}$, then, as an especial case, the rejection region of these tests would be more or less similar to the rejection region of Theorems 4.3 and 4.4 in [13].

Example 2

Let $X_{1}, X_{2}, \ldots, X_{n}$ be a random sample from a population with the Uniform probability density function

$$
f_{\theta}(x)=\frac{1}{\theta} ; 0<x<\theta
$$


According to Theorem 3, a UMP test of size $\alpha$ for testing the hypothesis $H_{0}: \theta_{1} \leq \theta \leq \theta_{2}$ versus $H_{1}: \theta<$ $\theta_{1}$ or $\theta>\theta_{2}$ is obtained as

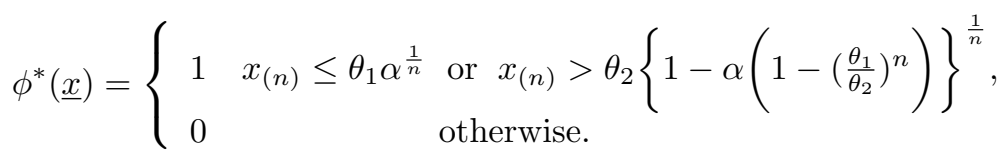

It should be mentioned that, if $\theta_{1}=\theta_{2}=\theta_{0}$, then $\phi^{*}$ is coincided with the UMP test for testing simple hypothesis $H_{0}: \theta=\theta_{0}$ versus two-sided hypothesis $H_{1}: \theta \neq \theta_{0}$ (see [7], pp. 92).

The power function of the above test is given by

$$
\begin{aligned}
& \pi_{\phi^{*}}(\theta)=P_{\theta}\left(X_{(n)}<\theta_{1} \alpha^{\frac{1}{n}}\right)+P_{\theta}\left(X_{(n)}>\theta_{2}\left\{1-\alpha\left(1-\left(\frac{\theta_{1}}{\theta_{2}}\right)^{n}\right)\right\}^{\frac{1}{n}}\right) \\
& = \begin{cases}1 & \theta<\theta_{1} \alpha^{\frac{1}{n}}, \\
\alpha\left(\frac{\theta_{1}}{\theta}\right)^{n} & \theta_{1} \alpha^{\frac{1}{n}} \leq \theta<\theta_{2}\left\{1-\alpha\left(1-\left(\frac{\theta_{1}}{\theta_{2}}\right)^{n}\right)\right\}^{\frac{1}{n}}, \\
1-(1-\alpha)\left(\frac{\theta_{2}}{\theta}\right)^{n} & \theta \geq \theta_{2}\left\{1-\alpha\left(1-\left(\frac{\theta_{1}}{\theta_{2}}\right)^{n}\right)\right\}^{\frac{1}{n}} .\end{cases}
\end{aligned}
$$

Figure 1 shows the curves of $\pi_{\phi^{*}}(\theta)$ for the cases $\theta_{1}=3, \theta_{2}=4, n=5$ and $n=20$, for two usual significance levels $\alpha=0.05$ and $\alpha=0.01$.

\section{Conclusion}

The problem of testing hypothesis in the family with support dependent on the unknown parameter was considered. The GLR test and UMP test were obtained for testing a simple versus two-sided hypotheses in the considered family. The extension of the obtained results to other family of distributions, such as the Pitman family, is a potential work for future research.

\section{REFERENCES}

1. R. Aaberge, and L.C. Zhang, A class of exact UMP unbiased tests for conditional symmetry in small-sample square contingency tables, Journal of Applied Statistics, vol. 32, pp. 333-339, 2005.

2. D. Birkes, Generalized likelihood ratio tests and uniformly most powerful tests, The American Statistician, vol. 44, pp. 163-166, 1990.

3. A. Birnbaum, Admissible test for the mean of a rectangular distribution, The Annals of Mathematical Statistics, vol. 25, pp. $157-161,1954$

4. D. Edelman, A note on uniformly most powerful two-sided tests, The American Statistician, vol. 44, pp. 219-220, 1990.

5. D.G. Kabe, and A.G. Laurent, On some nuisance parameter free uniformly most powerful tests, Biometrical Journal, vol. 23, pp. 245-250, 1981.

6. E.L. Lehmann, and C. Stein, Most powerful tests of composite hypotheses I. normal distribution, The Annals of Mathematical Statistics, vol. 19, pp. 495-516, 1948.

7. E.L. Lehmann, and J.P. Romano, Testing Statistical Hypotheses, Third Ed., Springer, New York, 2005.

8. P. Majerski, and Z. Szkutnik, Approximations to most powerful invariant tests for multinormality against some irregular alternatives, TEST, vol. 19, pp. 113-130, 2010.

9. M.P. McDermott, and Y. Wang, Construction of uniformly more powerful tests for hypotheses about linear inequalities, Journal of Statistical Planning and Inference, vol. 107, pp. 207-217, 2002.

10. S. Migliorati, Uniformly most powerful tests for two-sided hypotheses, Metron, vol. LX-N, pp. 107-114, 2002.

11. K. Nomakuchi, A note on the uniformly most powerful tests in the presence of nuisance parameters, Annals of the Institute of Statistical Mathematics, vol. 44, pp. 141-145, 1992.

12. J.W. Pratt, Admissible one-sided tests for the mean of a rectangular distribution, The Annals of Mathematical Statistics, vol. 29, pp. 1268-1271, 1958

13. A. Sayyareh, G. Barmalzan, and A. Haidari, Two sided uniformly most powerful test for Pitman family, Applied Mathematical Sciences, vol. 74, pp. 3649-3660, 2011. 
14. H. Scherb, Determination of uniformly most powerful tests in discrete sample spaces, Metrika, vol. 53, pp. 71-84, 2001.

15. K. Takeuchi, A note on the test for the location parameter of an exponential distribution, The Annals of Mathematical Statistics, vol. 40, pp. 1838-1839, 1969.

16. Y. Wang, L.J. Young, and D.E. Johnson, A UMPU test for comparing means of two negative binomial distributions, Communications in Statistics - Simulation and Computation, vol. 30, pp. 1053-1075, 2001. 